\title{
CONSTRAINT-BASED AND FUZZY LOGIC STUDENT MODELING FOR ARABIC GRAMMAR
}

\author{
Nabila A. Khodeir \\ Informatics Department, Electronic Research Institute, Cairo, Egypt
}

\begin{abstract}
Computer-Assisted Language Learning (CALL) are computer-based tutoring systems that deal with linguistic skills. Adding intelligence in such systems is mainly based on using Natural Language Processing (NLP) tools to diagnose student errors, especially in language grammar. However, most such systems do not consider the modeling of student competence in linguistic skills, especially for the Arabic language. In this paper, we will deal with basic grammar concepts of the Arabic language taught for the fourth grade of the elementary school in Egypt. This is through Arabic Grammar Trainer (AGTrainer) which is an Intelligent CALL. The implemented system (AGTrainer) trains the students through different questions that deal with the different concepts and have different difficulty levels. Constraint-based student modeling (CBSM) technique is used as a short-term student model. CBSM is used to define in small grain level the different grammar skills through the defined skill structures. The main contribution of this paper is the hierarchal representation of the system's basic grammar skills as domain knowledge. That representation is used as a mechanism for efficiently checking constraints to model the student knowledge and diagnose the student errors and identify their cause. In addition, satisfying constraints and the number of trails the student takes for answering each question and fuzzy logic decision system are used to determine the student learning level for each lesson as a long-term model. The results of the evaluation showed the system's effectiveness in learning in addition to the satisfaction of students and teachers with its features and abilities.
\end{abstract}

\section{KEYWORDS}

Language Tutoring Systems, Student Model, Constraint-Based Modeling, Fuzzy logic

\section{INTRODUCTION}

Computer-Assisted Language Learning (CALL) is a research discipline that concerned with using computers, new media, and information technologies for language learning [1], [2]. The integration of artificial intelligence and adaptive technologies into CALL is called Intelligent Computer-Assisted Language Learning (ICALL) [3]. Such technologies provide different adaptation dimensions to the language learning system. They are based on observing learners through system use and build an updating model of student knowledge and characteristics. Intelligent Tutoring Systems (ITSs), Adaptive Hypermedia systems (AHSs), Collaborative environments (CE), and computer-supported ubiquitous learning (CSUL) are the common adaptive approaches that have been used in ICALL [4]. The ITS approach is used in supporting the student problem-solving. That is through diagnosing the student answers and identifying the embedded errors and their origin. That allowing providing immediate and tailored feedback to the learner [5]. In addition, ITS approach is used in controlling the sequencing of learning items and/or tasks to reach a specific learning goal.

The focus of this paper is using ITS technology to support the student in solving grammar problems. AI techniques such as natural language processing (NLP), fuzzy logic, and student 
modeling techniques are used. Fuzzy logic incorporates precise techniques for talking vague information in the knowledge representation and decision making. It is one of the choices for appropriate evaluation of a learner's performance among the existing approaches such as neural, Baysian networks [6]. Karaci presented an example in using a fuzzy logic decision system to determine the learning level based on MYCIN certainty factor and the number of times the student takes for answering the question [6]. Another example presents developing parsers to identify grammatical errors by incorporating NLP techniques in analyzing student input [7]. Then the analyzed and interpreted observed student behaviors are mapped onto the student model. There are different student modeling techniques which are generally classified into two categories: cognitive science and machine learning approaches [8]. Cognitive Science approaches modeled how humans learn based on domain modeling and expert systems. Model-tracing (MT) and constraint-based modeling (CBM) are the two common techniques in the cognitive science approach. Model-tracing is based on using production rules, where rules that have been applied by the student with a high success rate are assumed to be learned [9]. This technique requires modeling of all alternative solutions and specifying each solution path using production rules [10][11].

Ohlsson proposed CBM as an approach to avoid some limitations of model-tracing, such as having runnable models of the expert and the student in addition to identifying and augmenting the student bugs. CBM is based on capturing the domain principles using a set of constraints. A constraint is represented by the tuple $\langle\mathrm{Cr}, \mathrm{Cs}\rangle$, where the relevance condition $\mathrm{Cr}$ is the condition under which the constraint is applicable, while the satisfaction condition Cs is the condition that should be satisfied such that the constraint is not violated [12][13]. Identifying errors, in this case, is based on tracking the violated constraints. In such a situation, the tutoring system can respond by giving advice to the student about the error, even without being able to replicate it.

Representing domain is typically achieved by breaking the domain knowledge into smaller elements (concepts) [14]. Neglecting relations between concepts lead to a set or vector model. Using such a model as a user model restricts updating techniques to only one concept or node at a time. Relations between domain concepts are considered in different manners. The network model is one of them where the items are arranged into a network and are interconnected by different types of links, specifically prerequisite links, is-a or part-of links, and remedial links. The hierarchy model is also considered in considering relations between concepts where the items are arranged to make a tree-like hierarchical structure. In such a model the more general items are placed higher in the hierarchy and are decomposed into simpler ones nearer to the bottom. The hierarchy model and the network model are the more appropriate models for grammar-oriented systems to define relations between items/terms as remedial or prerequisite [4]. Arabic is a Semitic native language of hundreds of millions of people worldwide, especially in the Middle East. Arabic morphology is considered to be one of the main elements that cause the language to be a very derivational and well-structured language [15]. Over the past decade, Arabic NLP (ANLP) has gained the interest of many researchers. Moreover, several systems have been developed for a multitude of applications for various purposes, including ICALLs [16] [17] This paper presents the design of an ICALL, for 4th grade Arabic grammar skills, that diagnoses students' answers and gives adaptive feedback to them. The domain knowledge is described as a hierarchical structure of grammatical skills, based on which a set of constraints in $(\mathrm{Cr}, \mathrm{Cs})$ form is described for constraint-based student modeling (CBSM). The main contribution is the proposed mechanism for efficiently checking constraints by utilizing the hierarchical structure of the domain knowledge. Such a technique facilitates diagnosing student errors and their origins. In addition, a fuzzy logic decision system is integrated to deal with uncertainty in the determination of the student learning level for each lesson. 
The rest of the paper is organized as follows: Section two presents related work to highlight the contributions of the paper. Section three and four give a brief demonstration of AGTrainer and its questions bank respectively. Section five addresses the domain hierarchal representation. Sections six, seven, and eight respectively explain student modeling, feedback, and student learning level in AGTrainer. Finally, sections nine and ten respectively present the evaluation and the conclusion.

\section{RELATED WORK}

ICALLs have been implemented for various languages including English, German, Chinese, and Arabic. Those systems vary in their capabilities, linguistic skills they focus on, the student modeling techniques, and the learning strategies. We address some of the prominent ICALL in the following subsections. We also discuss the state of the art in Arabic LTSs.

ICALL that based on intelligent tutoring system technology has the ability to diagnose students' answers and gives adaptive feedback to them.

Heift et al. present German Tutor which is an Intelligent Language Tutoring System that has been specifically designed for Web delivery [18]. The system can handle different possible errors through a series of progress bars attached to concepts from the learning domain. The NLP component of the system consists of a grammar and a parser which analyzes sentences from the student and detects grammatical and other errors. The feedback modules of the system then correlate the comprehensive output of the parser with an error-specific feedback message. Individualization and adaptively is achieved with techniques of learner modeling by keeping score across a number of error types, or, nodes such as grammar, vocabulary, punctuation, etc. According to the learner's model, the amount and wording of the feedback are adjusted.

Costa et al. present an integrated rule-based machine translation approach to disambiguate students' intended meaning in a CALL system [19]. They aimed to provide precise feedback to correct ungrammatical input by using Machine Translation technology to check the student's intended. Mal-rules are a specific kind of rules that extend a descriptive grammar to make it accept ungrammatical phenomena. These mal-rules are used both to identify grammar errors and to reconstruct the semantics of ungrammatical inputs which lead to triggering helpful messages to language learners The Student Model is the center of information. It contains vocabulary known by each student, the grammatical complexity the student is expected to work with, and the entire history of the student's interaction with the system such as previously completed exercises, previous mistakes, and time spent with each exercise.

Troussas et al. identify misconceptions, originating from neglect, lack of knowledge, or language transfer between simultaneous learning of multiple foreign languages. They present a model for misconception detection and identification (MDI) [20]. The MDI mechanism integrates the Fuzzy String Searching and The String Interpreting Resemblance algorithms in order to reason between possible learners' misconceptions. The system relays on a database with possible students' misconceptions. This database was constructed using anonymous learners' data which is served as training data for the proposed algorithm. They classify misconceptions into three types. The first error category is the spelling mistakes which can be the result of negligence or ignorance. The second category is the mistakes in the use of tenses and the third category is the mistakes in the use of auxiliary verbs. Fuzzy string searching is used to identify the first error category by detecting the pattern of strings that correspond to the possible answers that are stored in the system's database. On the other hand, the string interpreting resemblance techniques is used to identify spelling misconceptions due to language transfer and misconceptions in the use of tenses 
and auxiliary verbs. It tries to find a translation of the learner's given answer and compares it with the stored possible matches. The results of the error diagnosis are used to automatically model the learning process through four fuzzy sets to represent the student's knowledge level of each domain concept.

Several Arabic LTSs have been developed over the years. Tutoring Arabic grammar and syntax are the main focus of most of those systems. One of the earliest Arabic grammar tutoring systems included an expert system developed in Prolog for representing Arabic syntax using a set of production rules for detecting some syntax errors [21].

The more advanced Arabic ICALL system has been developed through a number of versions for tutoring the Arabic language to primary school students in Egypt in addition to foreigners learning Arabic as a second language [17] [22]. ICALL is based on NLP tools containing morphological and syntax analyzer to detect student errors. In addition, the system augmented the Arabic grammar with mal-rules that are capable of parsing ill-formed input when the grammatical rules fail. The main limitation of ICALL is the lack of a student model.

Shaalan et al. included a student model in a system for analysis and feedback of erroneous Arabic verbs [23]. The student learning skill levels are represented in terms of the frequency of an error that the student has made while satisfying its related concept. It is a form of a perturbation model. In addition, the system offers adaptive feedback for improving learner's linguistic knowledge according to the student level.

The main drawbacks of the presented systems are their dependency on pre-stored answers or malrules or database with possible students' misconceptions. This requires defining a list of possible errors in addition to using different mechanisms to correctly interpret a student's error when there is confusion with potential errors.

Generally the knowledge-based and corpus-based approaches are commonly used in ICALL [24]. The knowledge-based approach depends on morphological and grammatical rules that are written by linguists. Such an approach assists diagnosing learner errors and provides highly accurate feedback for these errors. That is usually based on defining a list of possible errors that are described as mal-rules.

On the other hand, the corpus-based approach utilizes learning algorithms that require large tagged learner corpus for training and testing to generate statistical models. Such a model can be used for learner's error prediction. The corpus-based approach is characterized by its adaptability in minimal time when sufficient large datasets are available which is not enough in the Arabic language.

We adopt knowledge-based in addition to utilizing a hierarchal representation of the system domain knowledge. Since Arabic linguistic grammar skills can be arranged hierarchically, and the relations between different skills can be defined. Another contribution is using such skill structure in addition to the CBM representation in diagnosing the students' errors. In other words, the hierarchical representation is used as a mechanism for efficiently checking constraints to model student knowledge and diagnosing student errors. In addition, that short student model, that represented by satisfied and unsatisfied constraints, is utilized with the trail number of solving the presented question to build a long-term model based on a fuzzy logic decision system. 


\section{Agtrainer System}

We developed AGTrainer which is an ICALL system [25][25]. It dedicated to training students at the primary level in Egyptian schools on Arabic language grammar. The system covers the basic concepts in Arabic grammar through a number of lessons. These lessons include demonstratives (الاشارة اسماء); parts of speech (words type), which include nouns (الاسماء), verbs (الإفعال), and

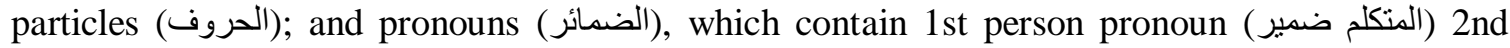
person pronoun (ضمير الرخاطب), and 3rd person pronoun (الغائب ضمير). Other lessons are deal with

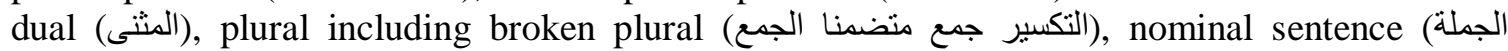
الإسمية), verbal sentence (الفعلية الجملة), noun of place (الككان ظرف (الزمان), noun of time (الجمان ظرف),

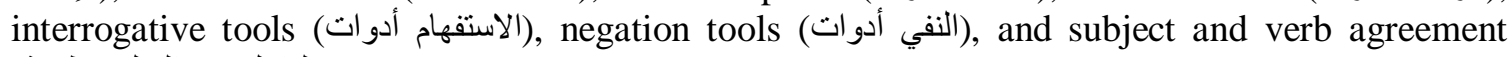
(الفاعل مع الفعل مطابقة). Those lessons are listed on the left-hand side of the system interface as shown in Figure 1 in the order with which they are taught to the students at the school. However, the student can choose any lesson without being bound by the order.

When a specific lesson is selected, different question types in the required grammatical skills will be offered successively. The student can proceed with the same question type by clicking "Next Question" or "Previous Question" buttons. Different types of questions can be selected using the "New Question" button.

The system responses to the student's answer by two levels of feedbacks. General feedback is presented to indicate if the question is correctly answered or not. Besides, informative feedback is given in case of totally or partially student wrong answer.

Another trail is then given to the student to correct his/her answer. Student can also go back to the related lesson that is explained through the tutor module by selecting the "Return to the lesson" button.

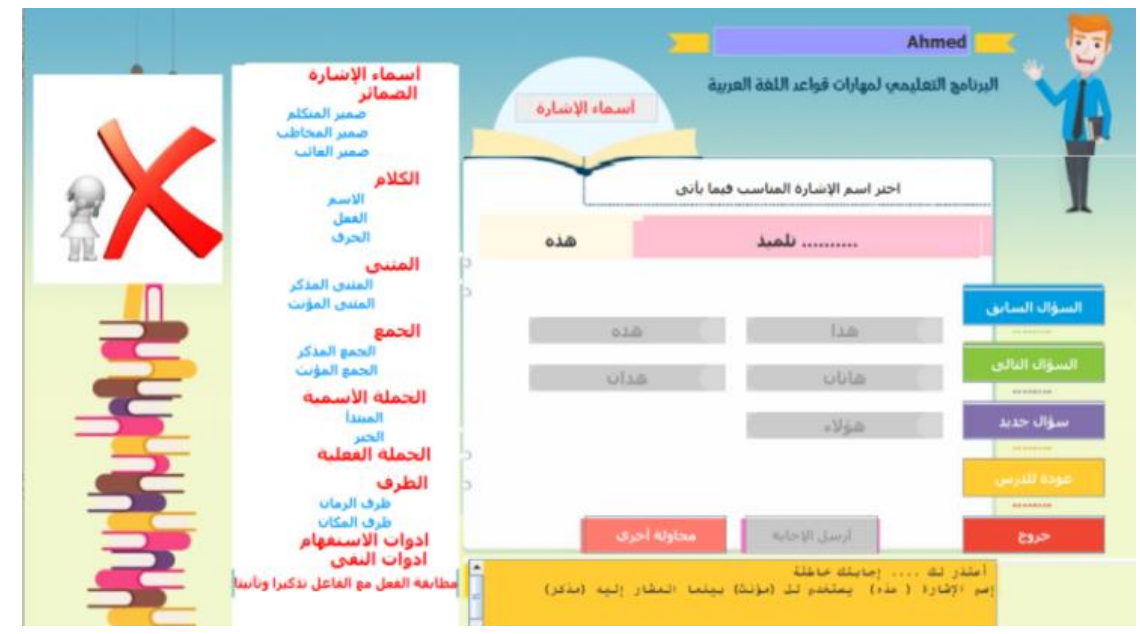

Figure 1. Screenshot of the AGTrainer Interface

\section{QUESTION BANK}

Questions bank is a database implied different types of questions that cover the listed lessons. For each lesson, all related grammatical concepts are addressed through different questions. For example; demonstrative lesson has questions that test the student's knowledge of different demonstratives, other questions consider agreement demonstrative with the noun it refers to in gender and number with considering exception in irrational plural. The degree of difficulty of the 
questions depends on the number of grammatical concepts to be addressed and whether the question is subject to exceptions to those concepts.

Each question in the database is defined by its header, statement, and available choices. The header defines the lesson it belongs to and the grammatical skills it addresses. The difficulty level of the header is based on the number of grammatical skills it tackles. The statement determines if the question is subject to grammatical exceptions or not which define the difficulty level of the statement.

\subsection{Question Forms}

There are two forms of the questions; selection-form for identifying one or more words that satisfy the required criteria(s) such as Fig 2 (a, b), or multiple-choice such as Fig. 2 (c, d). Some questions combine the two forms, for example, Fig. 2 (e, f). Although these forms do not allow the student to key in an Arabic sentence freely, they make the diagnosis of errors focused on correcting the basic concepts of this young student ages instead of being distracted in correcting spelling errors.

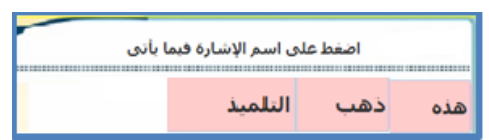

Figure 2(a)

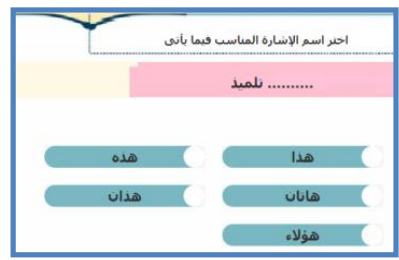

Figure 2(c)

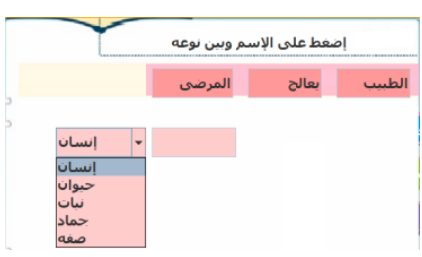

Figure 2(e)

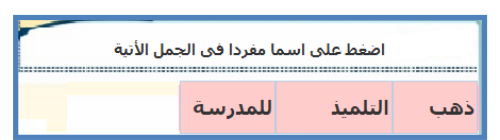

Figure 2(b)

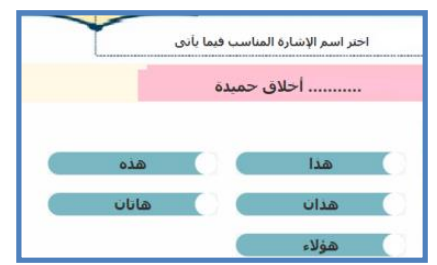

Figure 2(d)

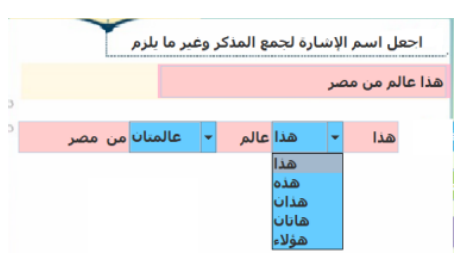

Figure 2(f)

\subsection{Questions Difficulty levels}

Questions are varying in their difficulty levels. The main parts of the question are an explanation of a question header, a given statement, and choices. Each question part contributes to defining the grammar concept the question addressed and the question difficulty level it has. For example; question in fig.2 (a) header is "يأتي فيما الإثارة إسم على أضغط" (Click on the demonstratives as follows") that addresses identifying word type and subsequently identifying noun subcategory. Such skills are used to characterize the noun and the subcategory of that noun. The question 
choices are "التلميذ ،ذهب ،هذه" ("This is, student, went") which are different in their type (noun and verb). So, the choices added another dimension which is the difference in word type.

Question 2 (c) and (d) have the same header " يأتي فيما المناسب الإشارة اسم أختر (Choose the appropriate demonstratives as follows) and choices which are demonstratives. The skill needed is the agreement of demonstrative with the noun it refers to in gender and number. The difference between the two questions is the given statement. The first given statement uses single masculine noun "student" while the next question uses irrational plural أخلاق "حميدة " (good ethics) which is subject to an exception in the needed skill. So, a further complication is added by the given statement.

Another example is the question (f) which has the header "يلزم ما وغير الدذكر للجمع الإشارة اسم أجعل" (change the demonstrative noun from the singular form into the plural form and change what is necessary to make it grammatically correct sentence.). One of the nouns in the given sentence has broken plural that does not follow the rule of conversion to plural.

\section{DOMAIN REPRESENTATION}

We consider Arabic basic grammar to be modeled in a hierarchal structure. For each domain concept or skill, there is a set of constraints that cover the corresponding grammar rules including the existing exception of each one. The application of these constraints shall take into account a defined hierarchical structure in addition to the possibility of applying them

\subsection{Arabic Basic Grammar}

The considered basic grammar rules expose to identifying word type and features, some morphological skills especially agreement between two words in different contexts, and a small part of the syntactic skill. The next sections will explore the considered skills and the hierarchy they implied. In addition, grain constraint representation for each skill will be explored through such representation.

Identifying word type and features are the basic Arabic grammar skill that taught at the early stage. It is a pre-request for many complicated grammatical skills and it is required in system grammatical lessons. Identifying word features starts by defining the word type if it is a noun or verb or particle. Based on the word type, more word features can be identified. For example, the verb tense is a feature associated with the verb word category. Other examples are identifying the noun and particle subcategories if the word category is noun or particle respectively. In other words, there is a hierarchy relationship between identifying word type and subcategories of type as shown in Figures 3. Such hierarchy facilitates the diagnosis of student errors and gives the possibility to know their underlying cause. For example, if the question asking about checking the verbs in the present tense and the student submit a wrong answer. Then we start by checking the word type to check if the student answer is a verb or not. If not, the student will be alerted to the basic error. Otherwise, the student answer (verb tens) will be tested.

Morphological skills are a science that discusses the change or conjugating of a word into various forms in order to achieve the desired meaning. Morphology is only applicable to nouns and verbs because they are able to be conjugated to different forms, unlike particles.

Morphology is applied to verbs by conjugating them from one tense to another or from one gender to another. On the other hand, it can be applied to nouns to conjugate them into the dual and plural forms. 
AGTrainer has two lessons "dual and plural" that dedicated to applying some of the morphological rules and changing the morphology of words to be inflected according to its role. In addition, broken plural is also considered. The remainder lessons expose to the morphological agreement between two words in different cases. Generally, many consecutive words need to be agreeing on one or two grammatical features with exceptions for some cases. That implies the dependency between words grammatical features and morphological agreement.

Syntactic skills refer to knowledge of grammar rules or the skills of constructing grammatically correct sentences in Arabic. Those skills include identifying word constituents according to certain syntactic features, identifying a sentence type, and converting from a given sentence type to another.

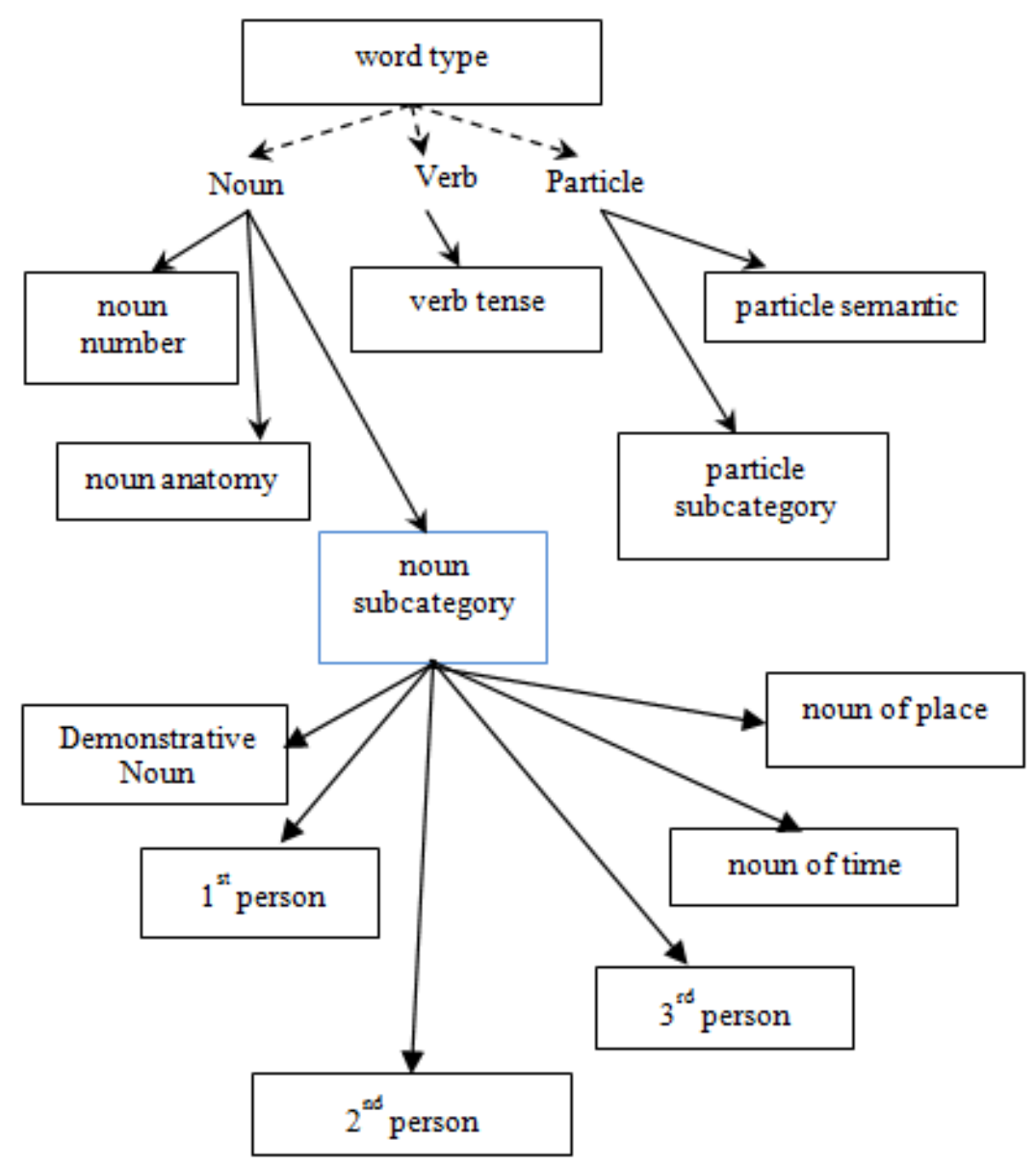

Figure 3. Identifying word grammatical features

\subsection{Constraint Based Modeling}

As we indicated in the last sections, we have three main skills: identifying word type and features, morphological and syntactic skills. Each skill is represented by a set of constraints with considering the different hierarchy levels. For example, figure 4 indicates part of constraints that represent Identifying masculine demonstrative noun. They are arranged according to the level it belongs to. As shown in fig. 5 constraints 15, 16, and 17 have different hierarchy levels. Constraint 15 checking if the selected answer is a noun. If this constraint is satisfied, the next constraint which has the same relevance condition and higher hierarchal level is checked. 
Constraint 16 checks the subcategory of the selected answer which is a noun. If constraint 16 is satisfied the next constraint with the highest hierarchal level is checked. It is worth to mention that, violation of a constraint blocking checking other constraints with the same relevance conditions and have higher hierarchy levels.

Another example is shown in figure 5 where some constraints of morphological agreement skill are indicated. That skill needs to another prerequisite skill where we have to ensure the student answer is a noun before we check the similarity of the features between the two words. Accordingly, the first constraint that deals with a case of the agreement (demonstrative with a word it refers to) defined as a prerequisite constraint that based on identifying word grammatical features skill with the first level that identifies if the word is a noun. Next constraints 36, 37 define agreement rules of gender and count. On the other hand, constraint 38 defines the exception of those constraints in case of irrational plural nouns.

\section{Identifying features constraints \\ Constraint 15:}

Cr: Identifying masculine demonstrative noun is required

Cs: the student answer should be a noun

\section{Constraint 16:}

$\mathrm{Cr}$ : Identifying masculine demonstrative noun is required

Cs: the student answer subcategory should be a demonstrative noun

\section{Constraint 17:}

$\mathrm{Cr}$ : Identifying masculine demonstrative noun is required

Cs: the student answer subcategory gender should be masculine

Figure 4. Identifying features constraints

\section{Agreement Constraints}

Constraint 35:

Cr: [Identifying noun], the skill is agreement and the student answer includes a demonstrative and a word it refers to

Cs: the word it refers to should be a noun

\section{Constraint 36:}

Cr: the skill is agreement and the student answer includes a demonstrative and the noun it refers to is sane

Cs: the gender of the noun should be similar to that of the demonstrative

\section{Constraint 37:}

Cr: the skill is agreement and the student answer includes a demonstrative and the noun it refers to is sane

Cs: the count of the noun should be similar to that of the demonstrative

\section{Constraint 38:}

Cr: the skill is agreement and the student answer includes a demonstrative and the noun it refers to is an irrational plural noun

Cs: the demonstrative should be هذه (singular feminine demonstrative)

Figure 5. Agreement constraints 


\section{Student Modeling}

The student modeling process aims to detect student mistakes and figure out their cause. This detection is achieved by matching each submitted student's answer against constraints with satisfied relevance conditions. If the student answer violates one or more constraints, the system supports the student with informative feedbacks that are attached to violated constraints.

Each question is defined by a set of grammar skills needed for correctly solving it. Accordingly, to check the submitted answer to a presented question, only the constraints corresponding to its skill set are checked. This speeds up the process and increases its efficiency. The student answer is correct if all the relevant constraints are satisfied. Errors are figured out based on the violated constraints.

Some questions need more than one skill to be correctly solved. Those skills can belong to the same grammar skills category or different skill categories. Moreover, the skills of the same category can belong to different levels of the skills hierarchy. This affects the sequence of constraints checking as explained in the following sub-sections.

\subsection{Different Levels in the Same Skill}

As explained earlier, skills in each category are arranged hierarchically. Constraints can exist at each level and each node in the hierarchy. For example, at the first level of the identifying word features skills hierarchy, three constraints check the word type (whether the word is a noun, a verb, or a particle). Assuming a given word is a noun, at the second level of the hierarchy, there are constraints to check the noun subcategory (whether the noun is a demonstrative, a pronoun (1st person, 2nd person, or 3rd person), a noun of place or a noun of time). It is worth mentioning that checking those constraints is blocked if checking the word category does not indicate a noun. In other words, checking some constraints is conditioned by the satisfaction of higher-level skill constraints. This speeds up the process and increases its efficiency. For example, the question in figure 2(a) that asks the student to click on the demonstratives noun, the correct answer is this هذا" since it is a demonstrative noun. If the student selects the verb go " ذهب ", the answer word category will be check if it is a verb. That will violate the higher-level constraint which leads to blocking the higher-level constraint to check the subcategory.

\subsection{Same Levels in the Same Skill}

In case the checked constraints belong to the same skill and at the same level in that skill hierarchy, all constraints should be checked. For example, referring to Figure 4, agreement in grammatical features such as count and gender are at the same level of the hierarchy. Where the student answer has to agree in both gender and count with the matched word in the question statement. Referring to the question in Figure 7, which asks the student to choose the appropriate demonstrative for nurses "همرضات" "هؤلاء", the correct answer is "these", since it agrees with "nurses" "مرضات in both gender (feminine) and count (plural). In that case, we must test all constraints with satisfied relevance conditions, regardless of whether or not one is satisfied or violated. The violation of one of the constraints does not block the testing of the rest, such as if the levels are different.

\subsection{Prerequisite Skill}

Some questions need skills from different categories to be correctly solved. Such constraints are arranged such that each constraint follows its prerequisite ones. For example, the question in 
figure 8 that asks the student to select a suitable noun that agrees with 2 nd person pronoun. The prerequisite condition for such a question is the selected word has to be a noun. This means applying the constraint of checking the word category to be sure it is a noun. If it is a noun the system will proceed in considering the agreement between the selected pronoun and the noun in the question statement. Accordingly, if checking the selected word category indicates that the student selected a word of a wrong category (verb or particle), AGTutor does not proceed to check the agreement conditions. That means violating the identifying word category constraints blocks checking the morphological skills constraints. This feature of AGTutor also speeds up the process and increases its accuracy in the diagnosing process.

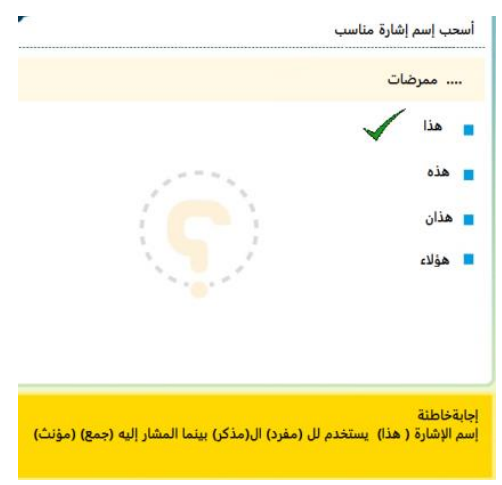

Figure 6. Applying constraints in the same hierarchically level in the same skill

\section{FeedBack Generation}

Different feedback strategies have been used for lexical and grammatical errors at the sentence level [26]. Providing the student with the correct solution is direct feedback while underlining his error(s) is indirect feedback. A metalinguistic explanation that providing grammar rules or the cause of the student errors is also considered. Recently more discursive forms of feedback such as providing model texts for the essay questions are addressed [27].

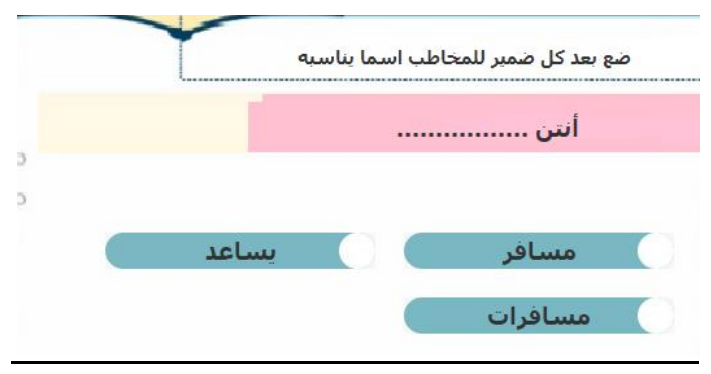

Figure 7. Question needs prerequisite skills

AGTrainer system supports the student through three types of feedback; general feedback which indicates the status of the student's answer if it is correct or wrong, direct corrective feedback (correct answer), and informative feedback. Informative feedback supports the student with diagnosing his/her error(s) and it is only generated if the student fails to submit the correct answer after two trials. Informative feedback could be considered as a metalinguistic or causal explanation. It is worth mentioning that, the system has not a runnable model to generate the correct answer as well as it does not store the questions model answers. So, checking and giving 
the general feedback of correct or wrong answer is based on satisfaction or violation of all or some constraints with satisfied relevance conditions.

Student answer has the potential to be partially correct which means a violation of some of the constraints with satisfied relevance conditions. So, we attach positive and negative feedback for each constraint to be presented in satisfaction and violation of that constraint. Positive feedback indicates the success of the application of the corresponding constraint and vice versa. The positive feedback is used only in the student's partial correct answer to indicate the correct part of the student answer. It is written in a way that allows the aggregation of messages that indicate the correct and wrong parts in the student answer. In addition, the stored constraints attached feedback messages include parameters that are substituted by the actual words mentioned in the question. That gives an adaptable message for each question.

For example, Identify demonstrative Noun constraint has the following feedback messages: Positive feedback:

$$
\text { (المختار المتكلم ضمير) المتكلم ضمير }
$$

1st person (selected 1st person)

\section{Negative feedback:}

منكلم ضمير وليست (المختار الإسم نوع) (المختارة الكلمة) كلمة

The word (selected word) is (word subcategory) and not a 1st person

Another example, Agreement 1st person pronoun with a noun in count constraint has the following feedback messages:

\section{Positive feedback:}

(المنكلم لضمير العدد في المناسب النوع) لل بيتخدم

is used for (correct selected 1 st person pronoun count)

\section{Negative feedback:}

(التالية الكلمة نوع) (إليها المشار الكلمة) الكلمة بينما (المختار المتكلم لضمير العدد في المناسب النوع) لل يستخدم

is used for (selected 1st person pronoun count) while (the word it refers to) is (count of the noun it refers to).

The word descriptions between brackets are replaced by the actual words in the question. Since checking satisfaction of each constraint requires retrieving the features of the student answer from system lexicon. That is used later in the substitution process.

In case of violation of some constraints and the satisfaction of other constraints, aggregation of positive feedback messages with negative feedback messages is presented. As shown in Figure 9 , the student is informed that he/she satisfied the selection of noun subcategory 1 st person as asked in the question and violated the agreement in count constraint where he selects plural 1st person pronoun while the consequent noun is a singular form of Arabic verb "like" أحب .

The messages before substitution:

\section{First message:}


(المختار المتكلم ضمير) المتكلم ضمير (selected

1 st person (selected 1 st person)

\section{Second message:}

(التالية الكلمة نوع) (إليها المشار الكلمة) الكلمة بينما (المختار المتكلم لضمير العدد في المناسب النوع) لل يستخدم

is used for (selected 1st person pronoun count) while (the word it refers to) is (count of the noun it refers to)

The messages after substitution:

First message: 1st person (we)

(نحن) المتكلم ضمير

\section{Second message:}

is used for (plural) while (like) is (singular)

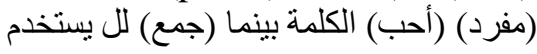

After substitutions the two messages are aggregated to be:

(مفرد) (أحب) الكلمة بينما (جمع) لل يستخدم (نحن) المتكلم ضمير

1st person (we) is used for (plural) while (like) is (singular)

The second example indicates the case of violation of more than one constraint, the aggregation of negative feedback messages is presented to the student as shown in Figure 7, where the student is informed that he/she violated the plural جمع and female مؤنث constraints. It is worth to mention that, in the aggregation process, we eliminate the repetition of words to produce a readable message.

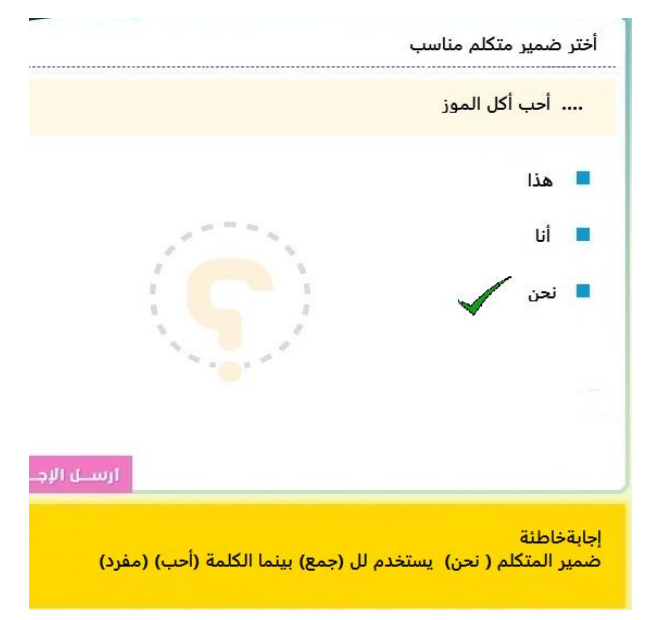

Figure 8. An example question with combined constraints

\section{Student Learning LeVel}


CBM represents the short-term student model where the assessment process is based on student response to a specific question. That model stores the satisfied constraints, violated constraints, and the number of trails for each question. To assess the learning level of the student in a specific lesson, we have to consider the student responses to all questions in that lesson. Karaci proposed ITS that is based on the overlay student model and CBM [6]. He considered each constraint as a separate problem and calculated MYCIN certainty factor for each one. That is based on grouping positive and negative points for constraint's satisfaction and violation respectively. In addition, he considered the trail number in estimating the learning level using a fuzzy logic decision system. Fuzzy logic was introduced by Zadeh and has been used in different fields implied educational grading [28]. A Fuzzy logic decision system is based on representing imprecise knowledge. It starts by fuzzifications of numerical inputs and mapping it to membership functions which is a range of inputs. This means the inputs are converted to verbal variables according to their respective values. Different forms of input membership functions are used such as; triangles, trapezoids, and Gaussian functions. Then some rules are proposed and evaluated using fuzzy reasoning. Then the result of the rules is combined and defuzzified.

In AGTrainer system we consider the satisfied constraints in addition to the number of trails to assess student's learning level. Moreover, the constraint's depth level in the hierarchal structure is also considered as an indication of the difficulty level of each constraint. We consider all these parameters in assessing the student learning level using fuzzy logic.

\subsection{Fuzzy logic input-output variables and fuzzy sets}

Two input variables are utilized in the fuzzy logic decision System; the constraints covering and trail numbers. The output variable is the learning level that represents a long-term student model. For each lesson, there is a set of constraints that must be satisfied in order for the student's learning level to be high. The same constraints can be used frequently with various questions. For this, we calculate each constraint satisfaction percentage compared to times the possibility of application.

To consider the hierarchal depth level in the long-term evaluation process, we set different satisfaction percentages for each constraint according to its hierarchal depth level. Satisfaction percentages are set to $70 \%, 60 \%, 50 \%$ for first, second, and third depth levels respectively. For constraints that deal with exceptions, the satisfaction percentage level is set to $40 \%$. These percentages are taken into account when calculating the number of constraints that were covered by the student in his solution to the problems.

The fuzzy sets for covering as an input variable are indicated in Fig. 3. It is an example of the first lesson that deals with demonstratives. Six constraints from different grammatical skills are required to check a student's answer to lesson questions. The fuzzy sets are defined as three trapezoids membership functions. The fuzzy sets and boundary values for the constraints covering the input variable are given in Fig. 9 and Table 1 respectively.

The second input is the trail number where more trail means that the student gets an explanation of his mistakes, which affects the calculation of his learning level. The fuzzy sets for the trail input variable and boundary values are shown in Fig. 10 and Table 2 respectively. The output variable is the learning level which is defined using four fuzzy which are indicated in Table 3

The inference rules to define the learning level in the AGTrainer system are shown in Table 4. All rules are evaluated in parallel using fuzzy reasoning. The most commonly used Mamdani's fuzzy inference mechanism (Mamdani, 1974) and the center of gravity (COG) defuzzification techniques are applied. 


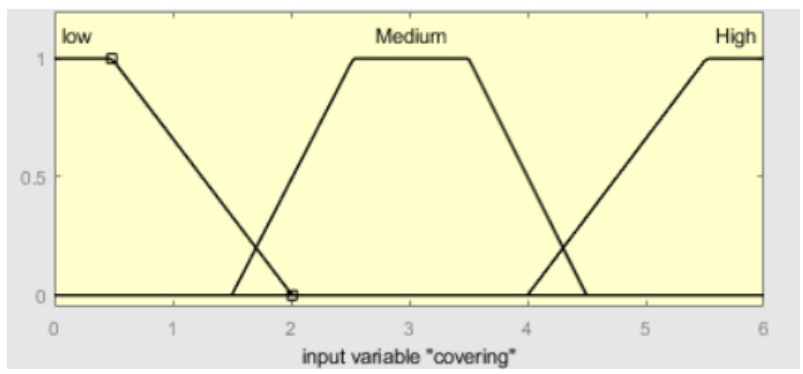

Figure 9. Fuzzy sets for covering input variable.

Table 1. Fuzzy sets and boundary values for Covering input variable

\begin{tabular}{cc}
\hline Fuzzy set & Limits \\
\hline Low & $0 \leq$ covering $\leq 2$ \\
Medium & $1.5 \leq$ covering $\leq 4.5$ \\
High & $4 \leq$ covering $\leq 6$ \\
\hline
\end{tabular}

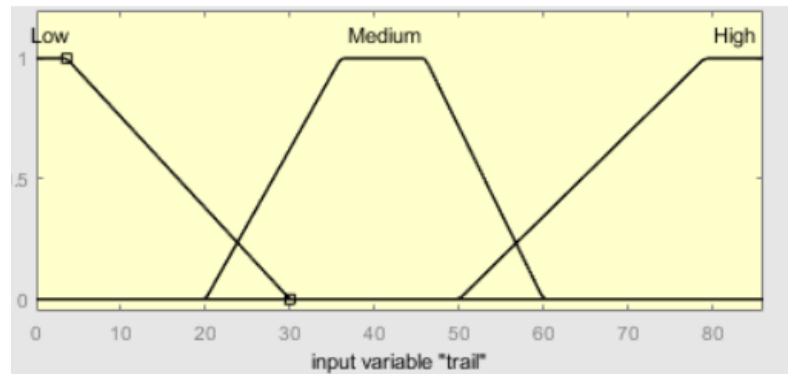

Figure. 10. Fuzzy sets for trail input variable.

Table 2. Fuzzy sets and boundary values for trail input variable

\begin{tabular}{cc}
\hline Fuzzy set & Limits \\
\hline Low & $0 \leq$ covering $\leq 30$ \\
Medium & $20 \leq$ covering $\leq 60$ \\
High & $50 \leq$ covering $\leq 86$ \\
\hline
\end{tabular}

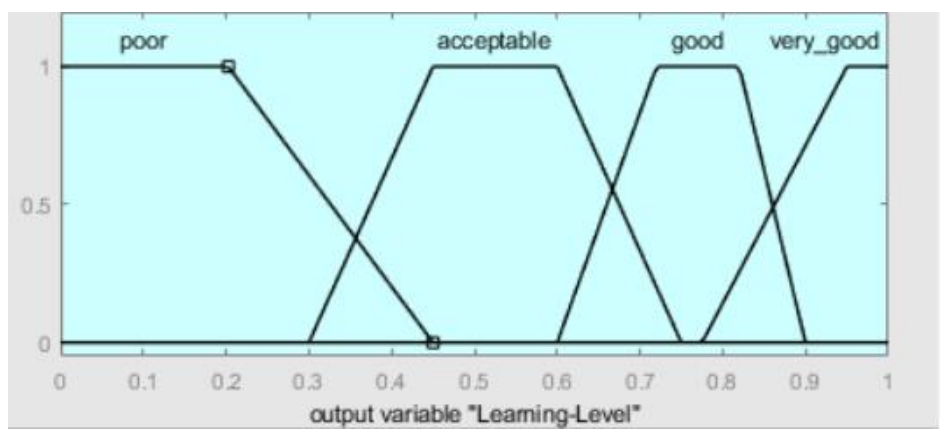

Figure. 11. Fuzzy sets for learning level output variable.

Table 3 Fuzzy sets and boundary values for Learning level output variable 
International Journal of Computer Science \& Information Technology (IJCSIT) Vol 12, No 4, August 2020

\begin{tabular}{cc}
\hline Fuzzy set & Limits \\
\hline Poor & $0 \leq$ covering $\leq \mathbf{0 . 4 5}$ \\
Acceptable & $0.3 \leq$ covering $\leq \mathbf{0 . 7 5}$ \\
Good & $0.6 \leq$ covering $\leq \mathbf{0 . 9}$ \\
Very-Good & $0.75 \leq$ covering $\leq 1$ \\
\hline
\end{tabular}

Table 4 Rule base used in the fuzzy logic decision system

\begin{tabular}{|c|c|c|c|}
\hline Trails Covering & \multirow{2}{*}{ Low } & Medium & High \\
\hline Low & \multirow{3}{*}{ Poor } & Good & Very-Good \\
\cline { 3 - 3 } & & Acceptable & \multirow{2}{*}{ Good } \\
\cline { 3 - 3 } Medium & Low & \\
\hline High & &
\end{tabular}

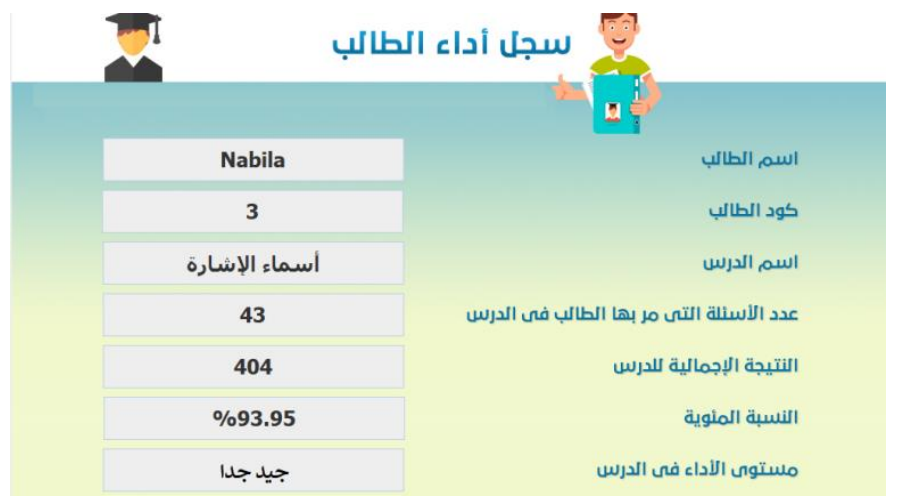

Fig. 12 System interface that indicates the student learning level

The output is the learning level that is presented to the student at the end of each lesson. Fig. 12 shows the system interface that indicates the student learning level in percentage which is $93.95 \%$ with the corresponding verbal value "very good".

\section{Evaluation}

The evaluation process targets the system effect, and satisfaction of the students and the teachers with AGTrainer. In addition, we conduct an experiment to get the learning level using AGTrainer with different inputs values for the fuzzy system.

\subsection{System Effect}

To assess the effect of AGTrainer in tutoring the students, 18 volunteer 4th-grade students at the primary level in Egypt were asked to get trained using the system as much as they wished for a period of two weeks. Their performance was recorded in terms of the violated and the satisfied constraints. For each student, we computed the average percentage of violated constraints the nth time each constraint was applicable. The global averages were then computed across all the students. The resulting power curve is depicted in Figure 13. The tutoring effect of AGTutor is obvious due to reducing the average percentage of violated constraints with learning. Power curves are increasingly used for evaluating ITSs provided that the assessed variable is represented by a set of constraints and that its value should be improved by learning [28].

\subsection{Satisfaction}


A satisfaction questionnaire formed of eight questions was prepared in order to assess the satisfaction of the students and the teachers with AGTrainer. We asked the respondents to provide their answers based on a Likert scale ranging from one to five, such that one refers to extreme dissatisfaction and five refers to the reverse. We computed the average for each respondent and then computed the global average. We obtained a value of 4.2 and 4.3 for the teachers and students respectively indicating a very high degree of satisfaction of both teachers and students with the capabilities and features of AGTrainer. We also confirmed the credibility of the questionnaire results using Cronbach's alpha as we obtained a value of $\alpha$ equal to 0.73 .

\subsection{Learning Level Experiment}

The percentages of satisfaction of each constraint are considered with the trail number to determine the learning levels for a number of students using the fuzzy logic decision system. Different covering and trail values are indicated in table 5. We consider the demonstratives lesson where there are six constraints with different depth levels and 43 questions with the possibility to repeat the attempt to answer each question twice. We can notice that when trail number increases the learning level decrees even the constraints coverage is high.

\section{Conclusions}

This paper presented AGTrainer, an ILTS for tutoring Arabic grammar skills to grade-four students at the primary stage in Egyptian schools. In AGTrainer, the basic different grammar skills are categorized and represented hierarchically within each category. Additionally, AGTrainer utilizes CBM to model domain knowledge using a set of constraints corresponding to the various skills. The hierarchical domain structure is used as a mechanism for efficiently checking constraints which facilitates diagnosing student errors and their origins. The system provides the student with parametrized feedback that indicates the correct and wrong parts of the student's answer.

The short-term student model is based on defining satisfied and violated constraints. The longterm model is proposed based on the short model information along with lesson interactions. The fuzzy logic decision system is integrated to deal with uncertainty in the determination of the student long term model or learning level model for each lesson. The system effect and satisfaction of the students and the teachers are considered in the evaluation process. In addition, an experiment to get the learning level is conducted based on different inputs values for the fuzzy system.

Each question in the database is defined by its header, statement, and available choices. The header defines the lesson it belongs to and the grammatical skills it addresses. The difficulty level of the header is based on the number of grammatical skills it tackles. The statement determines if the question is subject to grammatical exceptions or not which define the difficulty level of the statement. 
International Journal of Computer Science \& Information Technology (IJCSIT) Vol 12, No 4, August 2020

\begin{tabular}{lll}
\hline $\begin{array}{l}\text { Number of } \\
\text { covered } \\
\text { constraints }\end{array}$ & Tail number & $\begin{array}{l}\text { Learning } \\
\text { level }\end{array}$ \\
\hline 1 & 8 & 0.187 \\
1 & 46 & 0.187 \\
1 & 80 & 0.187 \\
\hline 2 & 8 & 0.75 \\
2 & 43 & 0.524 \\
\hline 2 & 80 & 0.2 \\
\hline 3 & 4 & 0.75 \\
\hline 3 & 49 & 0.52 \\
\hline 3 & 80 & 0.168 \\
\hline 4 & 4 & 0.76 \\
\hline 4 & 25 & 0.58 \\
4 & 80 & 0.22 \\
\hline 5 & 13 & 0.914 \\
\hline 5 & 44 & 0.75 \\
\hline 6 & 80 & 0.75 \\
\hline 6 & 12 & 0.915 \\
\hline 6 & 42 & 0.75 \\
\hline & 86 & 0.75 \\
\hline
\end{tabular}

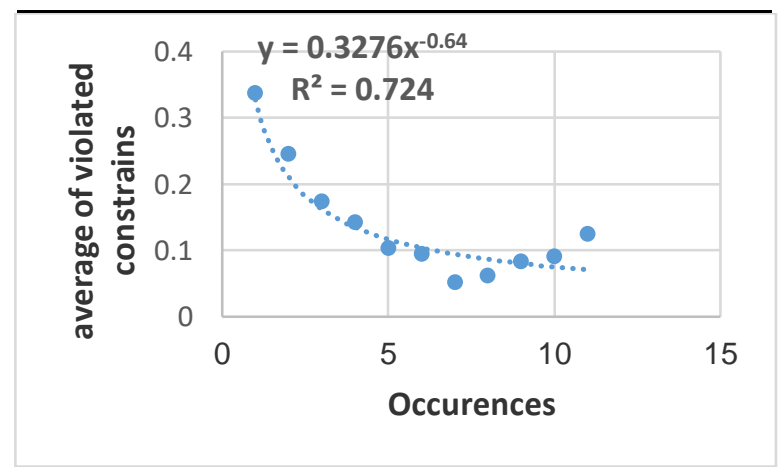

Fig. 13. Power curve depicting the tutoring effect of AGTrainer.

\section{REFERENCES}

[1] J. Lee, "Review of Language-learner computer interactions: Theory, methodology, and CALL applications.",’ Language Learning \& Technology, vol. 21, no. 3, pp. 72-75, 2017.

[2] L. Kohnke and H. Kong, "Review of New technologies and language learning," vol. 23, no. 1, pp. 36-39, 2019.

[3] J. Gamper and J. Knapp, “A Review of Intelligent CALL Systems," Computer Assisted Language Learning, vol. 15, no. 4, pp. 329-342, 2002.

[4] V. Slavuj, A. Meštrović, and B. Kovačić, “Adaptivity in educational systems for language learning: a review," Computer Assisted Language Learning, vol. 30, no. 1-2, pp. 64-90, 2017.

[5] S. Golonka, Ewa M and Bowles, Anita R and Frank, Victor M and Richardson, Dorna L and Freynik, "Technologies for foreign language learning: a review of technology types and their effectiveness," Computer assisted language learning, vol. 27, no. 1, pp. 70--105, 2014.

[6] A. Karaci, "Intelligent tutoring system model based on fuzzy logic and constraint-based student model," Neural Computing and Applications, vol. 31, no. 8, pp. 3619-3628, 2019.

[7] Heift, Trude. "Intelligent language tutoring systems for grammar practice." Zeitschrift für Interkulturellen Fremdsprachenunterricht 6.2, 2001.

[8] M. Feng, N. Heffernan, and K. Koedinger, "Student Modeling in an Intelligent Tutoring System," Intelligent Tutoring Systems in E-Learning Environments, no. May 2014, pp. 208-236, 2010. 
[9] P. Holt, S. Dubs, M. Jones, and J. Greer, “The State of Student Modelling," Student Modelling: The Key to Individualized Knowledge-Based Instruction, pp. 3-35, 1994.

[10] N.-T. Le and W. Menzel, "Using Weighted Constraints to Diagnose Errors in Logic Programming-The Case of an Ill-Defined Domain," International Journal of Artificial Intelligence in Education, vol. 19, no. 4, pp. 381-400, 2009.

[11] A. Mitrovic, K. R. Koedinger, and B. Martin, “A Comparative Analysis of Cognitive Tutoring and Constraint-Based Modeling," pp. 313-322, 2007.

[12] S. Ohlsson, "Constraint-Based Modeling: From Cognitive Theory to Computer Tutoring - And Back Again," International Journal of Artificial Intelligence in Education, vol. 26, no. 1, pp. 457-473, 2016.

[13] N. Khodeir, N. Wanas, and H. Elazhary, "Constraint-based student modeling in probability story problems with scaffolding techniques," International Journal of Emerging Technologies in Learning, vol. 13, no. 1, 2018.

[14] K. R. Roll, I., Baker, R. S., Aleven, V., McLaren, B. M., \& Koedinger, "User models for adaptive hypermedia and adaptive educational systems," User modeling- Springer Berlin Heidelberg, vol. 4321, pp. 367-376, 2005.

[15] A. Farghaly and K. Shaalan, "Arabic natural language processing: Challenges and solutions," ACM Transactions on Asian Language Information Processing, vol. 8, no. 4, pp. 1-19, 2009.

[16] K. Shaalan, S. Siddiqui, and M. Alkhatib, "Chapter 3," no. November, 2018.

[17] K. Shaalan, "An Intelligent Computer Assisted Language Learning System for An Intelligent Computer Assisted Language Learning System for Arabic Learners," Computer Assisted Language Learning, vol. 18, no. 1-2, pp. 81--109, 2005.

[18] T. Heift, "Web Delivery of Adaptive and Interactive Language Tutoring: Revisited," International Journal of Artificial Intelligence in Education, vol. 26, no. 1, pp. 489-503, 2016.

[19] L. da Costa, F. Bond, and X. He, "Syntactic Well-Formedness Diagnosis and Error-Based Coaching in Computer Assisted Language Learning using Machine Translation," Proceedings of the 3rd Workshop on Natural Language Processing Techniques for Educational Applications ( \{NLPTEA\}2016), pp. 107-116, 2016.

[20] C. Troussas, K. Chrysafiadi, and M. Virvou, “An intelligent adaptive fuzzy-based inference system for computer-assisted language learning," Expert Systems with Applications, vol. 127, pp. 85-96, 2019.

[21] S. Hegazi, N and Ali, G and Abed, EM and Hamada, "Arabic expert system for syntax education," in Proceedings of the Second Conference on Arabic Computational Linguistics, Kuwait, 1989, pp. 596-614.

[22] K. F. Shaalan and H. E. Talhami, "Arabic Error Feedback in an Online Arabic Learning System 1," pp. 203-212, 2006.

[23] K. Shaalan, M. Magdy, A. L. Y. Fahmy, K. Shaalan, and M. Magdy, "Analysis and feedback of erroneous Arabic verbs," Natural Language Engineering, vol. 21, no. 2, pp. 271-323, 2013.

[24] Ç. Eroglu et al., "Intelligent Natural Language Processing: Trends and Applications," vol. 740, no. 2, pp. 380-392, 2018.

[25] N. A. Khodeir, M. Hafez, H.Alazhary, "Multi-level Skills-based Student Modeling in an Arabic Grammar Tutor," System, IEEE\#41458-ACCS'017\&PEIT'017, Alexandria, Egypt, 2017.

[26] Guirao, Josefa Cánovas, Julio Roca de Larios, and Yvette Coyle. "The use of models as a written feedback technique with young EFL learners." System 52 (2015): 63-77.

[27] Bo, Yuan, ed. Fuzzy Sets, Fuzzy Logic, and Fuzzy Systems: Selected Papers by Lotfi A Zadeh. Vol. 6. World Scientific, 1996.

\section{AUTHORS}

Nabila Khodeir is a researcher in the Informatics department at the Electronics Research Institute, Cairo, Egypt. Her research interests include intelligent tutoring systems, user modelling and natural language processing. She earned her $\mathrm{Ph} . \mathrm{D}$. and ME from the Electronics and communications department at Cairo University. 